\title{
Health Insurance Coverage AND AdVERSE SELECTION
}

Philippe Lambert, Sergio Perelman, Pierre Pestieau, Jérôme Schoenmaeckers 


\title{
20 Health Insurance Coverage and Adverse Selection
}

\author{
Philippe Lambert, Sergio Perelman, Pierre Pestieau and Jérôme Schoenmaeckers
}

\subsection{Adverse selection and health insurance}

The term adverse selection is used in the insurance literature to describe a situation where an individual's demand for insurance (either the propensity to buy insurance, or the quantity purchased, or both) is positively correlated with the individual's risk of loss (e.g. higher risks buy more insurance), and the insurer is unable to allow for this correlation in the price of insurance. This may be because of private information known only to the individual, or because of regulations or social norms which prevent the insurer from using certain categories of known information to differentiate prices (e.g. the insurer may be prohibited from using information such as gender or ethnic origin or genetic tests). To test for the presence of adverse selection one checks the conjecture that contracts with more comprehensive coverage are chosen by agents with higher accident risk.

The problem is that such a test can as well reveal the presence of moral hazard. Like adverse selection moral hazard results from information asymmetry. In the case of adverse selection the informational issue concerns the individual's risk; in the case of moral hazard; it concerns the individual's behavior. Moral hazard occurs when the party with more information about its actions or intentions has a tendency or incentive to behave inappropriately from the perspective of the party with less information. Ex ante, it will be less cautious; ex post, it will seek overcompensation. In health insurance ex post moral hazard is likelier than ex ante moral hazard.

In the recent years, several authors have tested the relation between risk and insurance and have shown that the relation is not as clear as suspected. Chiappori and Salanié (1997, 2000) find no evidence of adverse selection in the automobile insurance market. Their main finding is that, although unobserved heterogeneity on risk is probably very important, there is no correlation between unobservable riskiness and contract choice. In other words, when choosing their automobile insurance contracts, individuals behave as though they had no better knowledge of their risk than insurance companies. They are the first to show that the riskcoverage correlation can be either sign and to stress the need of a new model. Similarly, in the life insurance market, Cawley and Philipson (1999) do not find evidence of adverse selection.

On the opposite side, Finkelstein and Poterba $(2004,2006)$ find evidence of adverse selection in the UK annuity market. Along the same lines, Olivella and 
Hernández (2006) observe the presence of adverse selection in British insurance markets, especially in private health insurance markets. Clearly the debate is wide open.

In this paper we intend to investigate on the basis of the information collected by SHARE if there is a relation, and if so what is its sign, between health risk and insurance coverage. In other words we are not planning to go beyond a simple statistical description of the relation between these two variables controlling for various characteristics of the concerned individuals.

For this purpose, SHARELIFE and wave 2 of SHARE contain valuable information. On the one hand, SHARELIFE surveyed retrospectively individuals about long periods of ill health or disability over their whole life. On the other hand, WAVE 2 questioned the same individuals on the characteristics of their health insurance coverage. Combining the answers given to these questions we estimate, for each country and for selected health care kinds, the relationship between health risks and insurance coverage, using a simple logistic model with full coverage as the dependent variable.

Two previous studies analyzed voluntary private health care insurance using SHARE data: (Paccagnella et al., 2008) and (Birò, 2010). They however have a different concern. Paccagnella et al. (2008) analyze the effect of having a voluntary health insurance policy on out-of-pocket spending for individuals aged 50 or more. They show that private insurance policy holders do not have lower out-ofpocket spending than the rest of the population. They also find that the main determinants of private insurance purchase are different in each country and this reflects the differences in the underlying health care systems. Birò (2010) is interested by the presence of moral hazard.

\subsection{Health insurance coverage}

In wave 2, individuals were asked the following question: Who finally pays for health care: yourself only, mostly yourself, mostly your health insurance, or your health insurance only? This question was repeated for several kinds of care: medical visits to doctors (general practitioners or specialists), hospitalization (in public or private hospitals), nursing care (at home or in nursing homes), as well as for dental care and prescription drug expenditures. In this study we are particularly interested in full health insurance coverage (paid by your health insurance only) for the first three kinds of care indicated above.

Figure 20.1 reports, for each country, the percentage of individuals reporting full health insurance coverage for visits to doctors, hospitalization and nursing care. In each case, we consider an individual to be fully covered if he/she reports full coverage for at least one of the kind of care considered, e.g., hospitalization in a public or a private hospital. The analysis is limited to the 50 to 79 years old individuals who participated in both, wave 2 and SHARELIFE. 
From Figure 20.1 and depending on the kind of care, SHARE countries can be classified according to the proportion of the aged population with full health insurance coverage. For visits to doctors and hospitalization coverage, a first group includes the Denmark (DK), The Netherlands (NL), Spain (ES), Italy (IT), Poland (PL) and Czech Republic (CZ), which reach rates close to or higher than $90 \%$; a second group is composed by Sweden (SE), Belgium (BE) and Switzerland $(\mathrm{CH})$ with percentages lower than $30 \%$; and finally, a third group comprises the rest of countries reporting intermediate rates of full coverage. For nursing care, the situation is dramatically different. In six countries - SE, BE, CH, AT, GR and CZ less than $20 \%$ of respondents reported full coverage.

As expected, this classification is highly driven by national health care insurance institutions.. As reported in Biró (2010), all SHARE countries analyzed here have universal mandatory health insurance, private for Switzerland but public for the others, the only exceptions are the Netherlands (NL) and Spain (ES) where high earners are excluded from public health insurance. But universal coverage does not mean full coverage, in several cases cost sharing is the rule. Moreover, it happens that people can not take a private insurance to cover their participation in health costs, e.g. Belgium for visits to doctors, subscribing to a private insurance for health expenditures not covered by social protection schemes (co-payment) is forbidden; and in others this is allowed, e.g. Germany, where a fixed co-payment amount, $10 €$, is charged in every quarter a doctor is visited (independent of total consumption).

Nevertheless, out of specific institutional regulations, in most cases full coverage is likely to be the result of a private individual decision. This is the main assumption we are making here. We postulate that individuals' health insurance behaviour is revealed by full coverage, compared with lower levels of coverage, and potentially affected by health risk expectations, as well as by other factors like gender, age, education and economic status. Another assumption is that respondents did not make systematic mistakes in reporting their health coverage status. Given the health insurance complexity, a potential measurement error bias exists which is probably related to individuals past experience with health providers and health insurance issues.

Figure 20.1: Health care full insurance coverage (\%) 


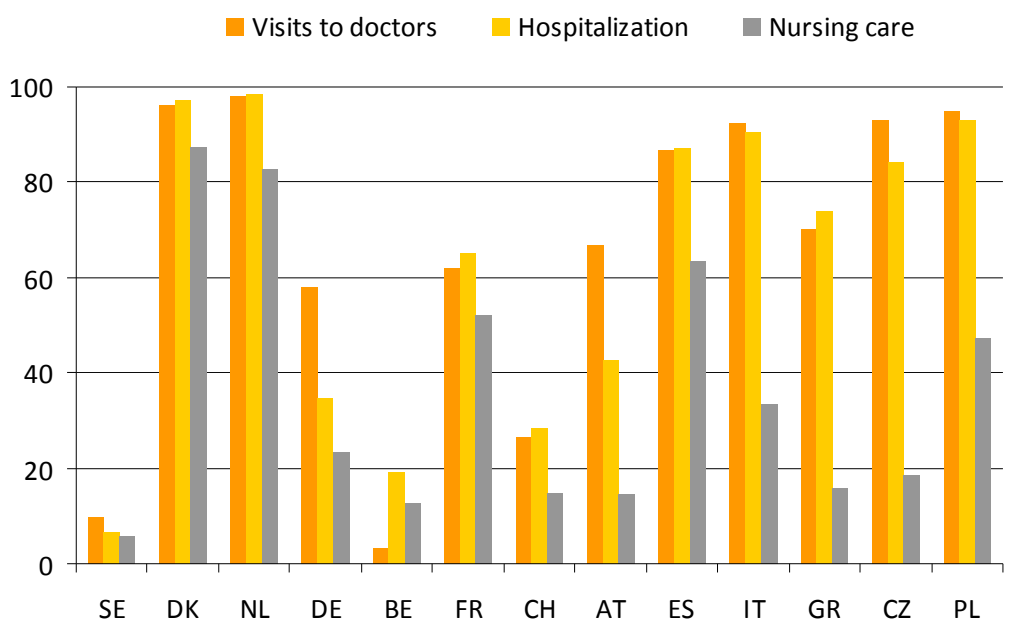

\subsection{A health risk indicator}

SHARELIFE adds a valuable retrospective dimension to SHARE, particularly on health status. For the purpose at stake in this study, the availability of retrospective information opens the possibility to build innovative indicators of individuals' health risks based on their own health status experience over previous periods of their life. By construction, these indicators would likely be more suitable than those built on the basis of contemporaneous information exclusively. We argue here that individuals' perception of health risks, a latent variable, is correlated with past health experiences which potentially affected individuals' health insurance behaviour.

Our choice of this indicator relies on the number of long periods of ill health in adulthood. They are reported by SHARELIFE respondents who were invited to answer the following question: Apart from any injuries you've already told us about today, as an adult, how many periods of ill health or disability have you had that lasted for more than a year: none, one, two, three or more, have been ill or with disabilities for all or most of my life?

From the original answers to this question, we computed the percentage of individuals who suffered one or two or more long term spells of ill health (the remaining category corresponds to individuals with no spells of ill health). Figure 2 reports the average country percentages. The two extreme cases are, on the one hand, Switzerland $(\mathrm{CH})$, with the lowest share of individuals with spells of ill health and, on the other hand, the Czech Republic (CZ), with nearly one third of the 50-79 cohort reporting at least one long term spell of ill health over their life 
span. Note that the variability of this indicator is high within countries across age and educational categories (not reported here).

Figure 20.2: Long-term periods of ill health (\%)

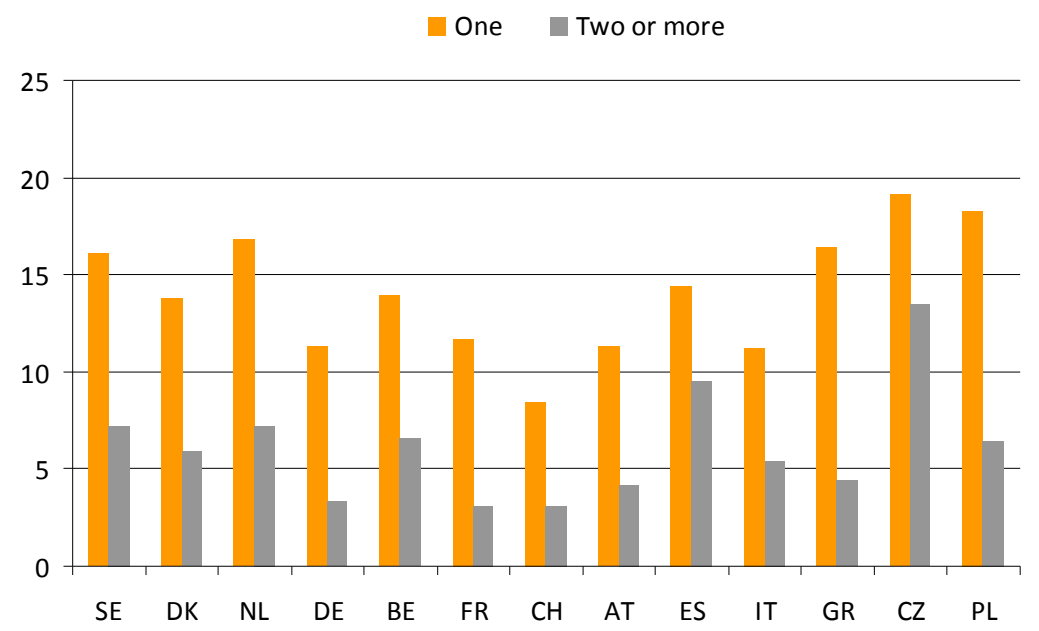

\subsection{The health risk insurance coverage correlation}

In order to test the potential correlation between health risks and health insurance coverage we proceed with the estimation of logistic models with full insurance coverage as the dependent variable. For each country, we estimate separately three models of full insurance coverage: visits to doctors, hospitalization and nursing care, respectively.

Table 20.1 reports the estimated parameters associated to the selected health risk indicator, which is the number of long-term ill health and disability periods, with values ranging from 0 (none) to 4 (have been ill or with disabilities for all or most of my life). The estimated parameters must be interpreted as marginal effects of increasing health risk on full health insurance coverage. A positive value would confirm the prevailing of individuals' adverse selection behaviour, and negative or statistically non significant parameters the absence of this relation. Several control variables were added to the model: age, gender, education and wealth quartiles.

Table 20.1: Logistic Model: Health risk variable parameter

$\begin{array}{llll}\text { Country } & \text { Visits to doctor } & \text { Hospitalization } & \text { Nursing care }\end{array}$




\begin{tabular}{|c|c|c|c|c|c|c|}
\hline & Parameter & (P-value) & Parameter & (P-value) & Parameter & (P-value) \\
\hline $\mathrm{AU}$ & 0.132 & $(0.229)$ & 0.189 & $(0.047)^{* * *}$ & 0.106 & $(0.378)$ \\
\hline $\mathrm{BE}$ & 0.072 & $(0.551)$ & -0.137 & $(0.056)^{*}$ & 0.128 & $(0.050)^{* * *}$ \\
\hline $\mathrm{CH}$ & -0.101 & $(0.462)$ & -0.109 & $(0.422)$ & 0.059 & $(0.715)$ \\
\hline $\mathrm{CZ}$ & -0.047 & $(0.635)$ & -0.001 & (0.988) & -0.201 & $(0.011)^{* * *}$ \\
\hline $\mathrm{DE}$ & 0.059 & $(0.456)$ & -0.039 & $(0.636)$ & 0.066 & $(0.459)$ \\
\hline DK & 0.310 & $(0.165)$ & 0.139 & $(0.521)$ & -0.147 & $(0.051)^{*}$ \\
\hline ES & 0.317 & $(0.011)^{* * *}$ & 0.313 & $(0.014)^{* *}$ & -0.094 & $(0.117)$ \\
\hline FR & 0.231 & $(0.011)^{* * *}$ & 0.255 & $(0.008)^{* * * *}$ & 0.246 & $(0.013)^{* *}$ \\
\hline GR & -0.130 & $(0.030)^{* *}$ & 0.014 & $(0.836)$ & -0.498 & $(0.001)^{* * * *}$ \\
\hline IT & 0.161 & $(0.230)$ & 0.545 & $(0.002)^{* * * *}$ & 0.096 & $(0.123)$ \\
\hline NL & -0.198 & $(0.266)$ & -0.416 & $(0.014)^{* *}$ & -0.097 & $(0.239)$ \\
\hline PL & 0.240 & $(0.205)$ & 0.223 & $(0.168)$ & -0.016 & $(0.823)$ \\
\hline $\mathrm{SE}$ & 0.141 & (0.127) & -0.042 & (0.779) & 0.187 & (0.148) \\
\hline
\end{tabular}

With the exception of France, no other country exhibits positive and significant parameters for the three health insurance branches analyzed here. However, we found also positive and significant parameters in the case of Spain (ES) for visits to doctors, in the case of Austria (AU), Spain (ES) and Italy (IT) for hospitalization and finally in the case of Belgium (BE) for nursing care. And these results appear to be driven mainly by health care institutions' regulations than by adverse selection behaviour. This is the case of France and Spain, for instance, where individuals with heavy diseases, like cancer, are fully reimbursed.

We proceed to some sensitivity analysis. First, similar results were obtained using alternative models in which the health risk indicator was represented by categories instead of a continuous variable. Second, also comparable results, but with even less significant parameters, were obtained with the sample restricted to the population aged 50 to 64 years old in order to limit the potential selectivity bias due to higher mortality rates among people in bad health. Third, separate regressions were performed by gender and the results generally confirmed those reported in Table 20.1, with the only exception of full hospitalization coverage in the case of men, for which near all the parameters were not significant. We also tried to explain our results using the main characteristics of national health care systems, which are regularly published by the OECD (2009). Nothing significant resulted from this exercise.

\subsection{Conclusions}

Summing up, it appears that with a few exceptions there is no evidence of a huge adverse selection problem in health insurance among European elderly people. 
And even the exceptions are likely driven by full public health coverage offered by some European countries for specific risks. Nevertheless, both adverse selection and moral hazard are a key issue to be taken into account in the design of health care insurance, mainly in the ongoing debate on long-term care insurance, public or private, in many countries. This has lead governments and insurance companies to offer lump-sum reimbursement and not full reimbursement in case of dependence. Such an arrangement is clearly unsatisfactory but can only be explained by adverse selection and ex post moral hazard, which are more pervasive in long term care than in acute health care.

Certainly this short paper offers very preliminary results that need to be confirmed when new waves of SHARE are available. In the meantime, what clearly appears from this exercise is that SHARELIFE and SHARE data combined offer a rich framework for future research on adverse selection in health insurance.

\section{References}

Bíró, A. (2010), Voluntary private health insurance and health care utilization of people aged 50+, Central European University, Budapest, mimeo.

Cawley, J. and T. Philipson, 1999. An empirical Examination of Information Barriers to Trade in Insurance. American Economic Review, Vol. 90, pp. 827-846.

Chiappori, P.A. and B. Salanié. 2000. Testing or Asymmetric Information in Insurance Markets. Journal of Political Economy, Vol. 108, pp. 56-79.

Chiappori, P.A. and B. Salanié. 1997. Empirical Contract Theory: The Case of Insurance Data. European Economic Review, Vol. 41, pp. 943-950.

Finkelstein, A. and J. Poterba. 2004. Adverse Selection in Insurance Markets: Policyholder evidence from the UK Annuity Market. Journal of Political Economy, Vol. 112, pp. 183-208.

Finkelstein, A. and J. Poterba. 2006. Testing for Adverse Selection with Unused Observables. National Bureau of Economic Research WP 12112.

OECD (2009), Health at a Glance 2009 - OECD Indicators, Paris.

Olivella P. and M. Vera Hernández. 2006. Testing for adverse selection into private medical insurance. The Institute For Fiscal Studies WP06/02.

Paccagnella, O., Rebba, V. and G. Weber. 2008. Voluntary private health care insurance among the over fifties in Europe: A comparative analysis of SHARE data. Department of Economics "Marco Fanno" WP 86, University of Padua. 